\title{
Assessment the association between liver cancer incidence and mortality rate with human development index in the European countries in 2012
}

\section{Mujtaba Shuja ${ }^{1,2}$, Sarah Islamie Farsani ${ }^{3}$, Hamid Salehiniya ${ }^{4}$, Salman Khazaei $^{5}$, Mahdi Mohammadian' ${ }^{6}$, Mohammad Aryaie7, Pezhman Bagheri $^{8}$, Fatemeh Allah Bakeshei ${ }^{9}$, Abdollah Mohammadian- Hafshejani $^{10,11, *}$}

*For correspondence:

amohamadii1361@gmail.com

Competing interests: The authors declare that no competing interests exist.

Received: 20 December 2016 Accepted: 27 February 2017 Published: 20 March 2017

Copyright The Author(s) 2017. This article is published with open access by BioMedPress (BMP).

This article is distributed under the terms of the Creative Commons Attribution License (CC-BY 4.0) which permits any use, distribution, and reproduction in any medium, provided the original author(s) and the source are credited.
${ }^{1}$ Health Promotion Research Center, Zahedan University of Medical Sciences, Zahedan, Iran

${ }^{2}$ School of Public Health, Iran University of Medical Sciences, Tehran, Iran

${ }^{3}$ Student's Research Committee, Shahrekord University of Medical Sciences, Shahrekord, Iran

${ }^{4}$ Zabol University of Medical Sciences, Zabol, Iran

${ }^{5}$ Department of Epidemiology, School of Public Health, Hamadan University of Medical Sciences, Hamadan, Iran

${ }^{6}$ Faculty of Nursing and Midwifery, Dezful University of Medical Sciences, Dezful, Iran

${ }^{7}$ Deputy of Research, Golestan University of Medical Sciences, Gorgan, Iran

${ }^{8}$ Non Communicable Diseases Research Center, Fasa University of Medical Sciences,

Fasa, Iran

9 University of Social Welfare and Rehabilitation Sciences, Tehran, Iran

${ }^{10}$ Department of Epidemiology and Biostatistics, Isfahan University of Medical Sciences, Isfahan, Iran

${ }^{11}$ Department of Epidemiology and Biostatistics, School of Public Health, Tehran University of Medical Sciences, Tehran, Iran 


\begin{abstract}
Background: Liver Cancer (LC) is one of the most common cancers in the worldwide. This cancer is considered as the fifth most common cancer in male and the ninth most common cancer in female. However, socioeconomic factors and morbidity and mortality of cancer are linked by sophisticated and flexible pathways. We were investigated the association between incidence and mortality of LC with the Human Development Index (HDI) in European countries in 2012. Methods: This study was an ecologic study in European countries for assessment the correlation between Age-Specific Incidence Rate (ASIR) and Age-Specific Mortality Rate (ASMR) of LC with HDI and its details including: Life expectancy at birth, Mean years of schooling and Gross National Income (GNI) per capita. We used of Pearson correlation method for appraisement the association between $\mathrm{HDI}$ and its components with ASIR and ASMR. Data of study was analyzed by SPSS15 statistical analysis software; the significance level of the tests was considered $P<0.05$. Results: Generally in 2012, European countries have recorded 63,462 new cases of LC, crude rate was 8.6 and ASIR was 4.3 per 100,000. On the other hand in Europe countries in 2012, 62,191 cases of deaths were occurred due to LC, crude rate was 8.4 and ASMR was 3.9 per 100,000. Strong positive Correlation was observed between ASIR and ASMR ( $r=0.848 ; P \leq 0.001)$. HDI have weak negative correlation with ASIR of LC ( $r$ $=-0.194 ; P=0.230)$, and strong negative correlation with ASMR of $L C(r=-0.515 ; P=$ 0.001). Conclusion: Increase in the human development index was associated with reduce in incidence and mortality of LC.
\end{abstract}

\title{
Keywords
}

Liver Cancer, HDI, Incidence, Mortality, Europe

\section{Introduction}

Liver cancer (LC) is one of the most common cancers in the worldwide (Wei et al., 2014). This cancer is considered as the fifth most common cancer in male and the ninth most common cancer in female (Hall and Wild, 2003). Estimates from the year 2012 indicate that 782,451 new cases of LC occurred worldwide, including 554,369 cases in male and 228,082 cases in female. Also in this year, 745533 case of death of LC were observed in worldwide, including 521,041 cases in male and 224,492 cases in female (Ferlay et al., 2014). This tumor accounted for $5.6 \%$ of all human cancers $(7.5 \%$ among male and $3.5 \%$ among female) in (Ferlay et al., 2001).

In the worldwide, the areas with high Age Standardized Incidence Rate (ASIR per $100,000)$ of LC are located in Eastern Asia (ASIR= 20.9), Intermediate rates 
happen in Africa $(A S I R=8.9)$ and Middle Africa America $(A S I R=8)$ and the lowest rates are in South-Central Asia $(A S I R=2.9)$ and Northern Europe $(A S I R=3.1)$ (Ferlay et al., 2014).

In the Europe, ASIR for LC in 2012 was equal to 4.3 per 100,000, with ranges from a relatively low rate in the Netherlands ( $A S I R=1.6)$ to the high rates in Republic of Moldova (ASIR=8.5). Also, Age Standardized Mortality Rate (ASMR per 100,000) for this cancer in the Europe in 2012 was 3.9 per 100,000, with ranges from a relatively low rate in Norway $(A S M R=1.7)$ to the high rates in Republic of Moldova (ASMR=9.5) (Mohammadian et al., 2015). LC is ordinarily a problem of the less developed countries (Ferlay et al., 2014). Extra than 85\% of LC new cases take place in developing countries which do not have suitable detection and treatment services (Ferlay et al., 2010). LC is the second most common cause of mortality from cancer in worldwide, estimated to be responsible for closely 746,000 deaths in 2012 (9.1\% of the whole death of cancer). The prognosis for LC is very low, so general ratio of LC mortality to incidence is around 0.95 (Ferlay et al., 2014).

Socioeconomic factors are connected with cancer incidence and mortality by intricate and variable pathways. One of the most important known meters of socioeconomic factors is Human Development Index (HDI). According to HDI, countries are assigned into four categories: countries with low $\mathrm{HDI}(\mathrm{HDI} \leq 0.5)$, countries with Medium HDI (0.8> $\mathrm{HDI}>0.5)$, countries with high $\mathrm{HDI}(\mathrm{HDI} \geq 0.8)$ and countries with very high $\mathrm{HDI}(\mathrm{HDI} \geq 0.9)$ (Bray et al., 2012).

In the study that conducted in Asian countries about relationship of incidence and mortality of LC with HDI, negative correlation was observed between ASIR and ASMR with HDI, But this relationships wasn't significant (Mohammadian et al., 2015). Similarly, relationship of HDI and incidence and mortality from other cancers were examined in other studies (Ghoncheh et al., 2015; HassanipourAzgomi et al., 2016; Mohammadian et al., 2015; Pakzad et al., 2015a, b; Pakzad et al., 2016; Pakzad et al., 2015c; Rafiemanesh et al., 2015). However, according to our information, no study has been done still regarding the association between the ASIR and ASMR of LC with HDI in European countries; therefore in this study we investigate the association between incidence and mortality of LC with HDI in European countries in 2012.

\section{Materials - Methods}

This study was an ecologic study in European countries for appraisal the relationship among Age-Specific Incidence Rate (ASIR) and Age-Specific Mortality Rate (ASMR) with Human Development Index (HDI) and its details including: Life expectancy at birth, average years of schooling and Gross National Income (GNI) per capita. Data about the ASIR and ASMR for every European countries for year 2012 had gathered from GLOBOCAN project that is 
available in (http://globocan.iarc.fr/Default. aspx) (Ferlay et al., 2015). Also data about Human Development Index had extracted from Human Development Report 2013 (Malik, 2013).

Details of Methods for estimate the Age-specific incidence rate (ASIR) and Agespecific mortality rate (ASMR) in GLOBOCAN project have been provided in previous reports (Ferlay et al., 2010; Ferlay et al., 2015; Foulkes and Cooney, 2010; Pakzad et al., 2015b; Pakzad et al., 2016).

\section{Statistical analysis}

In current report, we used of Pearson correlation method for assessment of correlation between Age-specific incidence rate and Age-specific mortality rate of LC by human development index and its details. All reported ASIR and ASMR were per 100,000 . Statistical significance was considered as $P<0.05$. All $P$-values reported in study are two-sided. Also, Statistical analyses were performed using SPSS (Version 16.0, SPSS Inc.).

\section{Results}

Overall, European countries have recorded 63,462 cases of LC, Crude Rate was 8.6 and ASIR was 4.3 per 100,000. The five countries with the highest ASIR of the LC were Republic of Moldova (ASIR=8.5), Italy (ASIR=7.1), Luxembourg $(A S I R=6.7)$, France $(A S I R=6.6)$, and Spain $(A S I R=5.9)$, respectively. Also, five countries with the lowest ASIR of the LC were The Netherlands (ASIR=1.6), Iceland $(A S I R=1.7)$, Norway $(A S I R=2.1)$, Belarus $(A S I R=2.1)$, and Ukraine $(A S I R=2.1)$, respectively.

On the other hand in Europe countries in 2012, 62,191 cases of deaths occurred due to LC, Crude Rate was 8.4 and ASMR was 3.9 per 100,000. The five countries with the highest ASMR of the LC were Republic of Moldova $(A S M R=9.5)$, Romania (ASMR=7.2), Montenegro (ASMR=6.6), Luxembourg $(A S M R=6.3)$ and Bosnia Herzegovina $(A S M R=6.2)$, respectively. Similarly, lowest $A S M R$ of the LC were in Norway $(A S M R=1.7)$, Belarus $(A S M R=1.9)$. The Netherlands $(A S M R=1.9)$, Iceland $(A S M R=2)$, and Ukraine $(A S M R=2.2)$, respectively. The countries with the highest and lowest ASIR and ASMR in both sexes are observable in Table 1. 


\section{Table 1. ASIR and ASMR of LC and HDI in Europe}

\begin{tabular}{|c|c|c|c|c|c|c|c|c|c|c|}
\hline \multirow[b]{2}{*}{ Country } & \multicolumn{3}{|c|}{ ASIR of LC } & \multicolumn{3}{|c|}{ ASMR of LC } & \multicolumn{4}{|c|}{ HDI and its details } \\
\hline & Total & Male & Female & Total & Male & Female & HDI & $\begin{array}{c}\text { Life } \\
\text { expectancy } \\
\text { at birth }\end{array}$ & $\begin{array}{c}\text { Mean } \\
\text { years of } \\
\text { schooling }\end{array}$ & GNI \\
\hline Albania & 4 & 5.1 & 2.9 & 5.7 & 7.2 & 4.2 & 0.749 & 77.1 & 10.4 & 7822 \\
\hline Austria & 5.2 & 8.1 & 2.8 & 4.7 & 7.2 & 2.6 & 0.895 & 81 & 10.8 & 36438 \\
\hline Belarus & 2.1 & 3.4 & 1.2 & 1.9 & 3.2 & 1 & 0.793 & 70.6 & 11.51 & 13385 \\
\hline Belgium & 3.1 & 4.7 & 1.7 & 2.9 & 4.2 & 1.8 & 0.897 & 80 & 10.9 & 33429 \\
\hline $\begin{array}{c}\text { Bosnia } \\
\text { Herzegovina }\end{array}$ & 4.6 & 5.8 & 3.7 & 6.2 & 8.1 & 4.7 & 0.735 & 75.8 & 8.3 & 7713 \\
\hline Bulgaria & 4.2 & 6.4 & 2.4 & 5.5 & 8 & 3.5 & 0.782 & 73.6 & 10.6 & 11474 \\
\hline Croatia & 4.7 & 8.1 & 2 & 4.5 & 7.1 & 2.4 & 0.805 & 76.8 & 9.8 & 15419 \\
\hline Cyprus & 2.7 & 3.8 & 1.7 & 2.4 & 3.5 & 1.4 & 0.848 & 79.8 & 9.8 & 23825 \\
\hline $\begin{array}{l}\text { Czech } \\
\text { Republic }\end{array}$ & 4.3 & 6.4 & 2.6 & 3.1 & 4.7 & 1.9 & 0.873 & 77.8 & 12.3 & 22067 \\
\hline Denmark & 2.9 & 4.5 & 1.4 & 2.6 & 4.3 & 1 & 0.901 & 79 & 11.4 & 33518 \\
\hline Estonia & 2.3 & 3.4 & 1.6 & 3 & 4.7 & 2 & 0.846 & 75 & 12 & 17402 \\
\hline Finland & 4.9 & 7.4 & 2.7 & 3.5 & 5 & 2.2 & 0.892 & 80.1 & 10.3 & 32510 \\
\hline France & 6.6 & 11.3 & 2.5 & 5.7 & 9.6 & 2.3 & 0.893 & 81.7 & 10.6 & 30277 \\
\hline $\begin{array}{c}\text { FYR } \\
\text { Macedonia }\end{array}$ & 4.1 & 5.7 & 2.6 & 5.5 & 7.5 & 3.8 & 0.59 & 69.6 & 5.6 & 3557 \\
\hline Germany & 4.6 & 7.2 & 2.3 & 3.6 & 5.4 & 2 & 0.92 & 80.6 & 12.2 & 35431 \\
\hline Greece & 3.4 & 5.3 & 1.8 & 4.5 & 6.9 & 2.5 & 0.86 & 80 & 10.1 & 20511 \\
\hline Hungary & 3.3 & 5.6 & 1.5 & 3.1 & 5.2 & 1.5 & 0.831 & 74.6 & 11.7 & 16088 \\
\hline Iceland & 1.7 & 2.1 & 1.3 & 2 & 2.6 & 1.5 & 0.906 & 81.9 & 10.4 & 29176 \\
\hline Ireland & 3.3 & 4.5 & 2.2 & 3 & 3.8 & 2.3 & 0.916 & 80.7 & 11.6 & 28671 \\
\hline Italy & 7.1 & 11 & 3.6 & 5.3 & 8.3 & 2.8 & 0.881 & 82 & 10.1 & 26158 \\
\hline Latvia & 3.3 & 5.6 & 1.8 & 2.7 & 4.3 & 1.6 & 0.814 & 73.6 & 11.5 & 14724 \\
\hline Lithuania & 2.7 & 4.7 & 1.3 & 2.7 & 4.5 & 1.6 & 0.818 & 72.5 & 10.9 & 16858 \\
\hline Luxembourg & 6.7 & 10.3 & 3.4 & 6.3 & 9.3 & 3.6 & 0.875 & 80.1 & 10.1 & 48285 \\
\hline Malta & 2.3 & 3.6 & 1 & 2.4 & 3.8 & 1.2 & 0.847 & 79.8 & 9.9 & 21184 \\
\hline Montenegro & 5.2 & 7 & 3.6 & 6.6 & 8.8 & 4.8 & 0.791 & 74.8 & 10.5 & 10471 \\
\hline Norway & 2.1 & 2.9 & 1.4 & 1.7 & 2.1 & 1.3 & 0.955 & 81.3 & 12.6 & 48 \\
\hline
\end{tabular}




\begin{tabular}{|c|c|c|c|c|c|c|c|c|c|c|}
\hline Poland & 2.8 & 3.8 & 2 & 2.8 & 3.7 & 2 & 0.821 & 76.3 & 10 & 17776 \\
\hline Portugal & 5 & 8.6 & 1.7 & 3.8 & 6.5 & 1.6 & 0.816 & 79.7 & 7.7 & 19907 \\
\hline $\begin{array}{c}\text { Republic of } \\
\text { Moldova }\end{array}$ & 8.5 & 12.5 & 5.4 & 9.5 & 14.1 & 5.9 & 0.66 & 69.6 & 9.7 & 3319 \\
\hline Romania & 5.8 & 9.2 & 3.1 & 7.2 & 11 & 4.2 & 0.786 & 74.2 & 10.4 & 11011 \\
\hline $\begin{array}{c}\text { Russian } \\
\text { Federation }\end{array}$ & 2.9 & 4.4 & 1.9 & 3.5 & 5.5 & 2.2 & 0.788 & 69.1 & 11.7 & 14461 \\
\hline Serbia & 4.5 & 6.6 & 2.7 & 4.7 & 6.7 & 3 & 0.769 & 74.7 & 10.2 & 9533 \\
\hline Slovakia & 4.3 & 6.8 & 2.4 & 3.6 & 5.6 & 2.2 & 0.84 & 75.6 & 11.6 & 19696 \\
\hline Slovenia & 4.9 & 8.4 & 2 & 4.1 & 6.8 & 1.9 & 0.892 & 79.5 & 11.7 & 23999 \\
\hline Spain & 5.9 & 9.9 & 2.4 & 4.3 & 6.9 & 2.1 & 0.885 & 81.6 & 10.4 & 25947 \\
\hline Sweden & 2.5 & 3.4 & 1.6 & 2.7 & 3.7 & 1.7 & 0.916 & 81.6 & 11.7 & 36143 \\
\hline Switzerland & 5.2 & 8.7 & 2 & 4 & 6.7 & 1.7 & 0.913 & 82.5 & 11 & 40527 \\
\hline $\begin{array}{l}\text { The } \\
\text { Netherlands }\end{array}$ & 1.6 & 2.4 & 0.9 & 1.9 & 2.6 & 1.2 & 0.921 & 80.8 & 11.6 & 37282 \\
\hline Ukraine & 2.1 & 3 & 1.4 & 2.2 & 3.2 & 1.5 & 0.74 & 68.8 & 11.3 & 6428 \\
\hline $\begin{array}{l}\text { United } \\
\text { Kingdom }\end{array}$ & 3.2 & 4.6 & 1.9 & 2.9 & 4.1 & 1.9 & 0.875 & 80.1 & 9.4 & 32538 \\
\hline
\end{tabular}

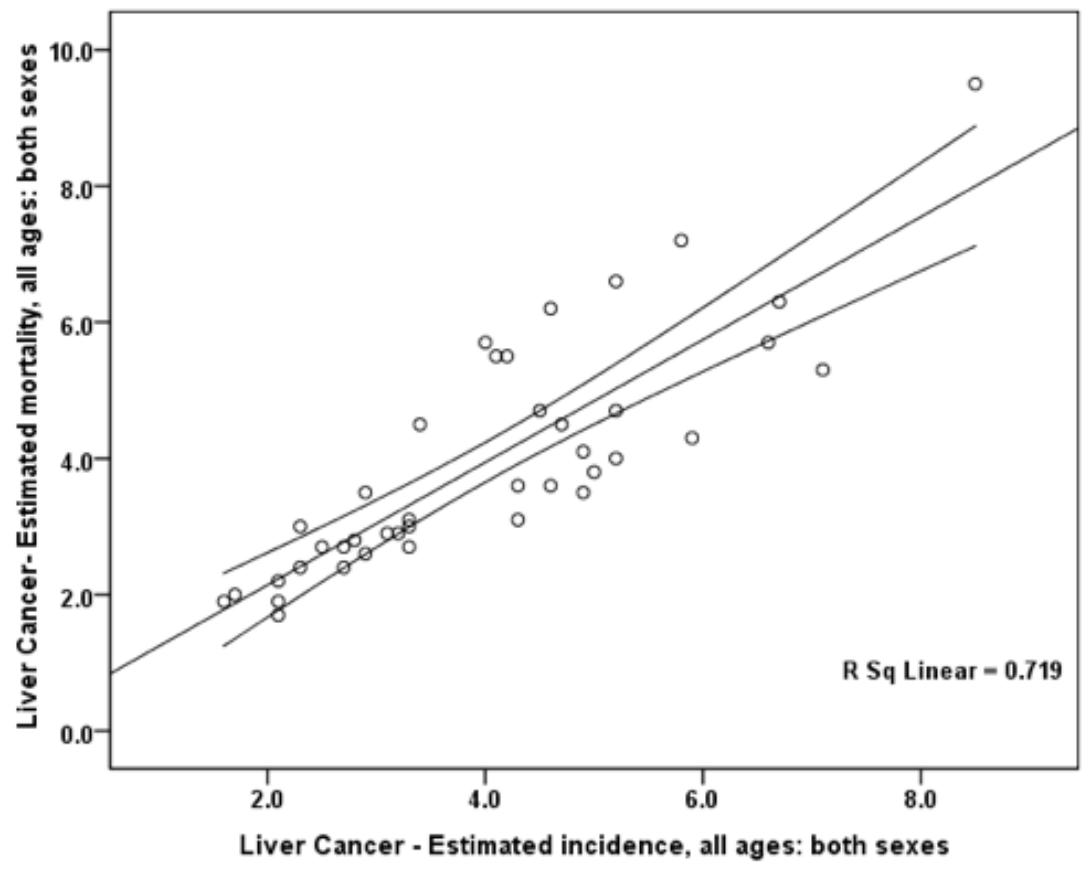

Figure 1. Correlation between ASIR and ASMR of LC in Europe in 2012. 


\section{ASIR and ASMR}

Between the Age-Specific Incidence Rate (ASIR) and Age-Specific Mortality Rate (ASMR) of LC, strong positive significant correlation was observed $(r=0.848 ; P \leq$ $0.001)$.Also, in males and females, strong positive significant correlation was observed between ASIR and ASMR of LC ( $r=0.850 ; p \leq 0.001)$ and $(r=0.897$; $P$ $\leq$ 0.001), respectively (Fig. 1).

\section{ASIR and HDI}

The weak negative correlation was observed between the HDI and ASIR of $L C(r=-0.194 ; P=0.230)$. Also, $A S I R$ have weak negative correlation with life expectancy at birth $(r=-0.064 ; P=0.695)$, mean years of schooling $(r=-0.290$; $P=0.070)$, and $G N I(r=0.059 ; P=0.718)$ (Fig. 2).

In male, a weak negative correlation was observed between the ASIR of LC and HDI ( $r=-0.114 ; P=0.482$ ). Also, ASIR have weak positive correlation with life expectancy at birth $(r=0.10 ; P=0.537)$, weak negative correlation with mean years of schooling $(r=-0.235 ; P=0.144)$, and weak positive correlation with $\mathrm{GNI}$ $(r=0.116 ; P=0.474)$.

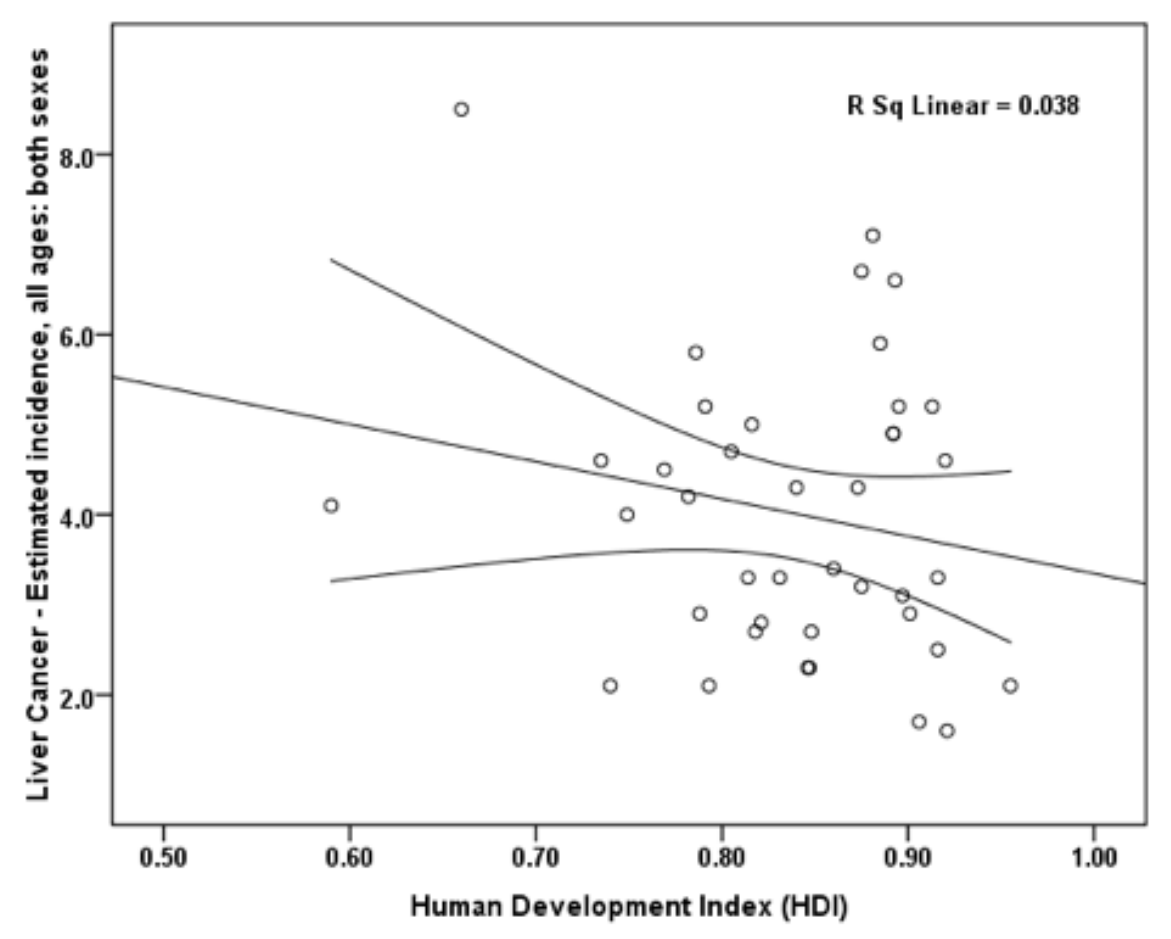

Figure 2. Correlation between HDI and ASIR of LC in Europe in 2012. 
In female, a strong negative correlation was observed between the ASIR of LC and $\mathrm{HDI}(r=-0.420 ; P=0.007)$. Also, ASIR have weak negative correlation with life expectancy at birth $(r=-0.164 ; P=0.312)$, and $G N I(r=-0.187 ; P=0.248)$, but has strong negative correlation with mean years of schooling $(r=-0.316 ; P=0.047)$.

\section{ASMR and HDI}

The strong negative significant correlation was observed between the ASMR of $\mathrm{LC}$ and $\mathrm{HDI}(\mathrm{r}=-0.515 ; \mathrm{P}=0.001)$. Also, ASIR have weak negative correlation with life expectancy at birth $(r=-0.221 ; P=0.171)$, and $G N I(r=-0.236 ; P=0.143)$, but has strong negative significant correlation with mean years of schooling $(r=-0.421 ; P=0.007)$, and (Fig. 3).

In male, a strong negative significant correlation was observed between the ASMR of LC and HDI ( $r=-0.456 ; P=0.003)$. Also, ASIR have weak negative correlation with life expectancy at birth $(r=-0.196 ; P=0.225)$, and $G N I$ ( $r=-0.176 ; P=0.276$ ), but has strong negative correlation with mean years of schooling ( $r=-0.380 ; P=0.016)$.

In female, a strong negative significant correlation was observed between the ASIR of LC and HDI $(r=-0.613 ; P \leq 0.001)$. Also, ASMR have strong negative significant correlation with life expectancy at birth ( $r=-0.331, P=0.037)$, mean years of schooling ( $r=-0.430 ; P=0.006)$, and $G N I(r=-0.381 ; P=0.015)$.

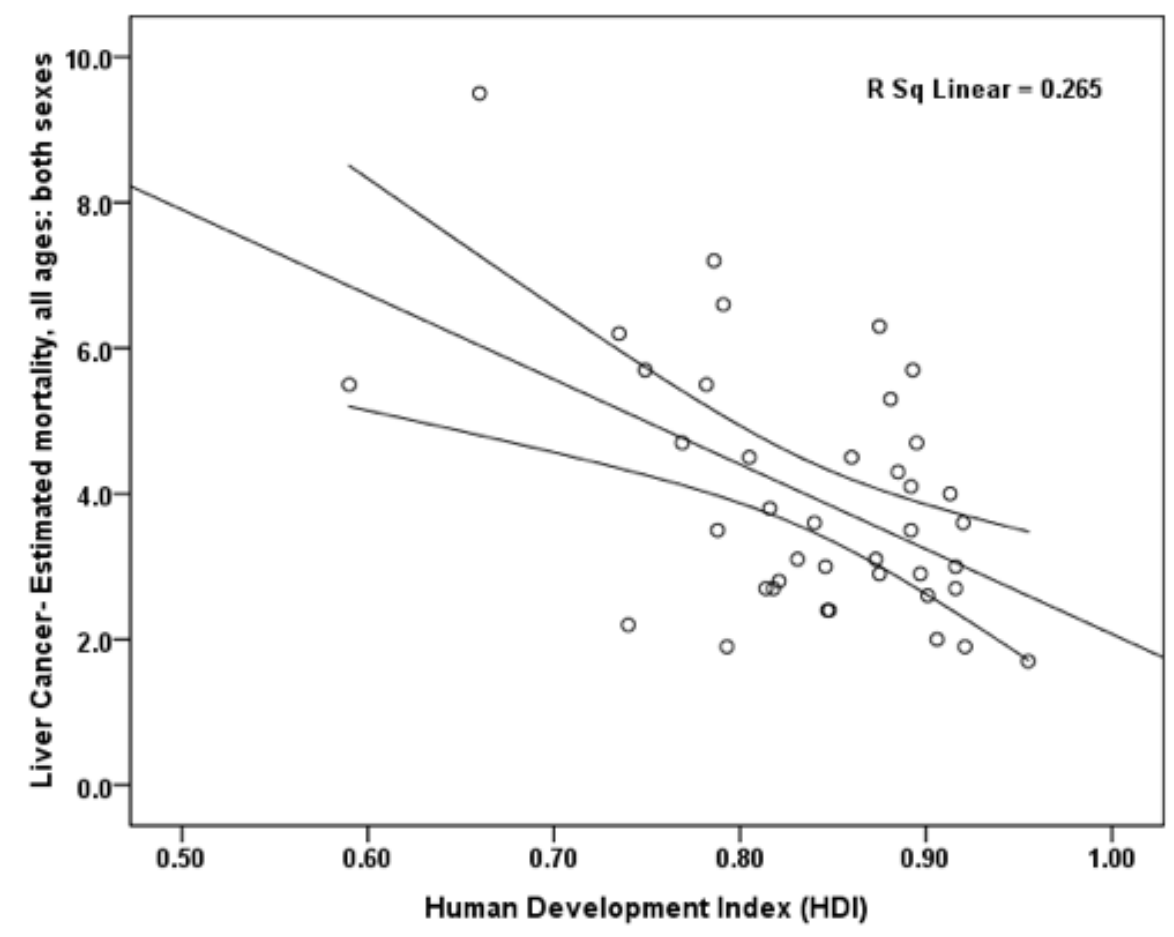

Figure 3. Correlation between HDI and ASMR of LC in Europe in 2012. 


\section{Discussion}

Overall in 2012, European countries have recorded 63,462 cases of LC, Crude Rate was 8.6 and ASIR was 4.3 per 100,000. On the other hand in Europe countries in 2012, 62,191 cases of deaths occurred due to LC, Crude Rate was 8.4 and ASMR was 3.9 per 100,000. Correlation between ASIR and ASMR was 0.848 ( $P \leq 0.001$ ), that was statistically significant. In this study between HDI and ASIR of LC a negative correlation was seen about $0.194(P=0.230)$, also between $\mathrm{HDI}$ and ASMR of LC was a negative correlation about $0.515(\mathrm{P}=0.001)$.

In European countries, Republic of Moldova ( $A S I R=8.5)$, Italy ( $A S I R=7.1$ ), Luxembourg (ASIR=6.7), France (ASIR=6.6, and Spain ( $A S I R=5.9)$, had the maximum ASIR of LC. Four of these countries were in very high level of $\mathrm{HDI}$, but Republic of Moldova was in Medium HDI level. In contrast, five countries with the lowest $A S I R$ of the $L C$ were The Netherlands ( $A S I R=1.6)$, Iceland (ASIR= 1.7), Norway (ASIR=2.1), Belarus (ASIR=2.1), and Ukraine ( $A S I R=2.1)$, respectively (Ferlay et al., 2015). The HDI in three countries were in very high level of HDI, but in two countries were in high level of HDI (Malik, 2013). The results of study that conducted with Chang and et al,. displayed that hepatocellular carcinoma by reason of chronic hepatitis $B$ infection was common in countries with low and medium level of HDI, mostly in Asian and African countries (Chang et al., 1997). However, the increased incidence of hepatocellular carcinoma in developed countries was mainly due to increase the prevalence of alcohol use and hepatitis $\mathrm{C}$ infection. It seems that the rise of $\mathrm{HCC}$ in western countries that have a higher $\mathrm{HDI}$, has been due to hepatitis $C$ infection and alcohol consumption (Liver, 2012). However, ASIR of LC in Europe in 2012 was 6.8 per 100,000 that was less than observed ASIR in the world (ASIR=15.3), Africa (ASIR=12.4), Asia (ASIR =20) and Oceania (ASIR=7.8) (Ferlay et al., 2015). In developing parts of the world, especially in Asian countries, cancer is becoming one of the most severe health problems. So that in some of these countries, such as Japan and South Korea, cancer is known as one of the most common causes of deaths. It is expected that if current management strategies and programs do not modified, the number of deaths from cancer by 2020 in these areas will reach 7.1 million cases per year (Mackay, 2006).

The distribution of cancers in different parts of the world in terms of human development index is very different, so in regions with very high, high, medium and low HDI, various cancers are classified as the most common cancer. cancer of the lung, breast, colorectal and prostate in areas with very high and high HDI are considered as the most common cancers that almost include half of new cancer cases in this region. Also, in regions with medium and low $\mathrm{HDI}$, in addition to breast, colorectal and lung cancer, cervical cancer, liver and stomach cancer classified as cancers with high incidence and mortality. However, in regions with low $\mathrm{HDI}$, cancers due to infectious agents included a large part of incidence and mortality of cancer (Bray et al., 2012; Franceschi and Wild, 2013). 
The results of this study presented that the ASMR of LC in Europe varies from 1.7 in Norway to 9.5 in Republic of Moldova. The highest ASMR of LC occurred in Republic of Moldova (ASMR=9.5), Romania (ASMR=7.2), Montenegro $(A S M R=6.6)$, Luxembourg $(A S M R=6.3)$ and Bosnia Herzegovina $(A S M R=6.2)$. In these countries only Luxembourg was in very high level of HDI. Similarly, five countries with lowest ASMR of LC were Norway (ASMR=1.7), Belarus $(A S M R=1.9)$, The Netherlands (ASMR=1.9), Iceland (ASMR=2), and Ukraine with $(A S M R=2.2)$ that were in very high and high level of $\mathrm{HDI}$.

This study is an ecological study and its results can be interpreted only at the population level and attribution of the results of this study to individual levels lead to occurrence of ecological fallacy. This study also has some advantages. The first advantage is that we studied all European countries. So the data have perfect integrity. The second advantage is the novelty of findings because we couldn't find a study that shows relationship between the ASIR and ASMR of LC with $\mathrm{HDI}$ and its components in European countries. Therefore, it is proposed that similar studies in other parts of the word should be done, so that the relationship hypothesis between the incidence and mortality rate of LC and HDI in these regions can be examined.

\title{
Conclusion
}

Correlation between ASIR and ASMR was statistically significant. Also, we were observed a negative correlation between $\mathrm{HDI}$ and the ASIR of LC, but between the HDI and ASMR of LC significant correlation was observed. Therefore, increase in the human development index was associated with reduce in incidence and mortality of LC.

\section{Abbreviations}

\author{
ASIR: Age-specific incidence rate \\ ASMR: Age-specific mortality rate \\ HDI: Human Development Index \\ LC: Liver Cancer
}




\section{Acknowledgements}

Hereby we appreciate of the cooperation of all employees involved in data collection in the GLOBOCAN project and World Bank.

\section{Author contribution}

All authors contributed to the design of the research. MS, SIF, SK and HS collected the data. HS, SK and AMH conducted analysis and interpretation of data. All authors drafted the first version. MM, MA, PB, FAB and AMH edited the first draft. All authors reviewed and commented on final draft. 


\section{References}

Bray, F., Jemal, A., Grey, N., Ferlay, J., and Forman, D. (2012). Global cancer transitions according to the Human Development Index (2008-2030): a population-based study. The lancet oncology 13, 790-801.

Chang, M.-H., Chen, C.-J., Lai, M.-S., Hsu, H.-M., Wu, T.-C., Kong, M.-S., Liang, D.-C., Shau, W.-Y., and Chen, D.-S. (1997). Universal hepatitis B vaccination in Taiwan and the incidence of hepatocellular carcinoma in children. New England Journal of Medicine $336,1855-1859$.

Ferlay, J., Bray, F., Pisani, P., and Parkin, D. (2001). Cancer incidence, mortality and prevalence worldwide, version 1.0. IARC CancerBase.

Ferlay, J., Shin, H.R., Bray, F., Forman, D., Mathers, C., and Parkin, D.M. (2010). Estimates of worldwide burden of cancer in 2008: GLOBOCAN 2008. International journal of cancer 127, 2893-2917.

Ferlay, J., Soerjomataram, I., Dikshit, R., Eser, S., Mathers, C., Rebelo, M., Parkin, D.M., Forman, D., and Bray, F. (2015). Cancer incidence and mortality worldwide: sources, methods and major patterns in GLOBOCAN 2012. International journal of cancer 136, E359-E386.

Ferlay, J., Soerjomataram, I., Ervik, M., Dikshit, R., Eser, S., Mathers, C., Rebelo, M., Parkin, D., Forman, D., and Bray, F. (2014). GLOBOCAN 2012 v1. 0, Cancer incidence and mortality worldwide: IARC CancerBase No. 11. 2013. International Agency for Research on Cancer Web site Available online: http://globocan iarc fr (accessed on 24 November 2014).

Foulkes, W.D., and Cooney, K.A. (2010). Male Reproductive Cancers (Springer).

Franceschi, S., and Wild, C.P. (2013). Meeting the global demands of epidemiologic transition-The indispensable role of cancer prevention. Molecular oncology 7, 1-13.

Ghoncheh, M., Mohammadian-Hafshejani, A., and Salehiniya, H. (2015). Incidence and mortality of breast cancer and their relationship to development in Asia. Asian Pac $J$ Cancer Prev 16, 6081-6087.

Hall, A.J., and Wild, C.P. (2003). Liver cancer in low and middle income countries. Bmj 326, 994-995.

Hassanipour-Azgomi, S., Mohammadian-Hafshejani, A., Ghoncheh, M., Towhidi, F., Jamehshorani, S., and Salehiniya, H. (2016). Incidence and mortality of prostate cancer and their relationship with the Human Development Index worldwide. Prostate International.

Mackay, J. (2006). The cancer atlas (Amer Cancer Society).

Malik, K. (2013). Human development report 2013. The rise of the south: Human progress in a diverse world. The Rise of the South: Human Progress in a Diverse World (March 15, 2013) UNDP-HDRO Human Development Reports.

Mohammadian, M., Soroush, A., Mohammadian-Hafshejani, A., Towhidi, F., Hadadian, F., and Salehiniya, H. (2015). Incidence and Mortality of Liver Cancer and Their Relationship with Development in Asia. Asian Pacific journal of cancer prevention: APJCP 17, 2041-2047.

Pakzad, R., Mohammadian-Hafshejani, A., Ghoncheh, M., Pakzad, I., and Salehiniya, H. (2015a). The incidence and mortality of lung cancer and their relationship to development in Asia. Translational lung cancer research 4, 763. 
Pakzad, R., Mohammadian-Hafshejani, A., Ghoncheh, M., Pakzad, I., and Salehiniya, H. (2015b). The incidence and mortality of prostate cancer and its relationship with development in Asia. Prostate international 3, 135-140.

Pakzad, R., Mohammadian-Hafshejani, A., Khosravi, B., Soltani, S., Pakzad, I., Mohammadian, M., Salehiniya, H., and Momenimovahed, Z. (2016). The incidence and mortality of esophageal cancer and their relationship to development in Asia. Annals of translational medicine 4.

Pakzad, R., Mohammadian-Hafshejani, A., Mohammadian, M., Pakzad, I., Safiri, S., Khazaei, S., and Salehiniya, H. (2015c). Incidence and Mortality of Bladder Cancer and their Relationship with Development in Asia. Asian Pac J Cancer Prev 16, 7365-7374.

Rafiemanesh, H., Mohammadian-Hafshejani, A., Ghoncheh, M., Sepehri, Z., Shamlou, R., Salehiniya, H., Towhidi, F., and Makhsosi, B. (2015). Incidence and Mortality of Colorectal Cancer and Relationships with the Human Development Index across the World. Asian Pacific journal of cancer prevention: APJCP 17, 2465-2473.

Wei, K.-R., Yu, X., Zheng, R.-S., Peng, X.-B., Zhang, S.-W., Ji, M.-F., Liang, Z.-H., Ou, Z.X., and Chen, W.-Q. (2014). Incidence and mortality of liver cancer in China, 2010. Chin J cancer 33, 388-394. 